\title{
Aeromagnetometria aplicada ao reconhecimento de feições estruturais e descontinuidades associadas a um batólito granítico - Caçapava do Sul, RS
}

Nicolas Rodrigues Hispagnol (UNIPAMPA/Campus Caçapava do Sul) e Maximilian Fries (UNIPAMPA/Campus Caçapava do Sul)

Copyright 2021, SBGf - Sociedade Brasileira de Geofísica

This paper was prepared for presentation during the $17^{\text {th }}$ International Congress of the Brazilian Geophysical Society held in Rio de Janeiro, Brazil, 16-19 August 2021.

Contents of this paper were reviewed by the Technical Committee of the $17^{\text {th }}$ International Congress of the Brazilian Geophysical Society and do not necessarily represent any position of the SBGf, its officers or members. Electronic reproduction or storage of any part of this paper for commercial purposes without the written consent of the Brazilian Geophysical Society is prohibited.

\section{Abstract}

The evolution, geometry and structural framework comprehension of intrusive granitic rocks is fundamental to enhance and provide subsidies of ore-related occurrences in the Sulriograndense shield, Rio Grande do Sul state, Brazil. This known and complex mineral province is formed in the Archean to neo/Paleoproterozoic presenting igneous, metamorphic, and sedimentary rocks. The present study main objective was to detect and delineate unknown surface. The present study main objective was to detected and delineat unknown surface mapped features. Such discontinuties and structures are related to structural and lithologic limits in the granitic batholith known as Suíte Granítica Caçapava do Sul (SGCS) (Caçapava do Sul Granitic Suite). Aeromagnetic data, processing and enhancement techniques was applied to delineate linear features and define magnetic domains. Additionally, Euler Deconvolution was performed in order to determine magnetic detected sources estimated depth. The results, were integrated to previous geologic basis (mapping) using GIS and allowed to improve and better understand the structures and framework in a deeper context. These preliminary contributions offers important subsidies in a regional-scale context. Provides, also, structures related detected anomalies for further geophysical local-scale surveys in order to maximize the understanding of the CGCS limits in depth and also nearby host rocks.

\section{Introdução e objetivos}

O Escudo Sul riograndense (ESRG) se caracteriza pelos seus conjuntos de rochas pré-Cambrianas e cambrianas, as quais compõem a parte sul da província Mantiqueira (Almeida \& Hasui, 1984). Segundo Hartmann et al. (2007) é constituído por rochas metamórficas, ígneas e sedimentares, vinculados aos estágios pré, sin e pós colisionais da orogênese Brasiliana no Neoproterozóico.

Esta região levanta diversos debates entre os geólogos por conta de sua dificuldade de se explicar a sua formação como uma possível continuação do Cráton pré-Brasiliano Rio de la Plata, no Rio Grande do Sul (que se estende até o complexo Luis Alves na área Setentrional) (Fragoso César, 1980, 1991, Brito Neves \& Cordani 1991) ou se são independentes.
Além de diversos debates geológicos sobre a formação do escudo, a sua importância econômica é reconhecida, pelas ocorrências de minérios de metais base e ouro nos municípios de Camaquã, Caçapava do Sul, Lavras do Sul e São Sepé. No ESRG os corpos intrusivos tardios possuem importante contribuição ao arcabouço estrutural da região e as ocorrências minerais associadas. Dentre estes batolitos, próximo ao município de Caçapava do Sul, encontram-se a Suíte Granítica Caçapava do Sul (SGCS) e o granito Santo Ferreira (GSF) (Nardi \& Bitencourt, 1989; Remus, 1999; e Ribeiro, 1970).

A SGCS, objetivo deste trabalho consiste uma feição extensa que apresenta cerca de $250 \mathrm{~km}^{2}$ de afloramento e, apesar dos mapeamentos realizados nas últimas décadas caracterizando a SGCS, há uma lacuna no conhecimento quanto a informações de subsuperfície como limites (laterais e em profundidade) e estruturas associadas. Considerando esses elementos acima descritos, este estudo tem como objetivo principal detectar e delimitar feições lineares por meio da aeromagnetometria na SGCS e arredores. Especificamente, propô-se identificar feições em subsuperfície e padrões estruturais associados a domínios magnéticos (direções preferenciais). Tais feições, conforme estudos prévios, apontam para uma relação direta com as ocorrências minerais conhecidas na região (Figura 1).

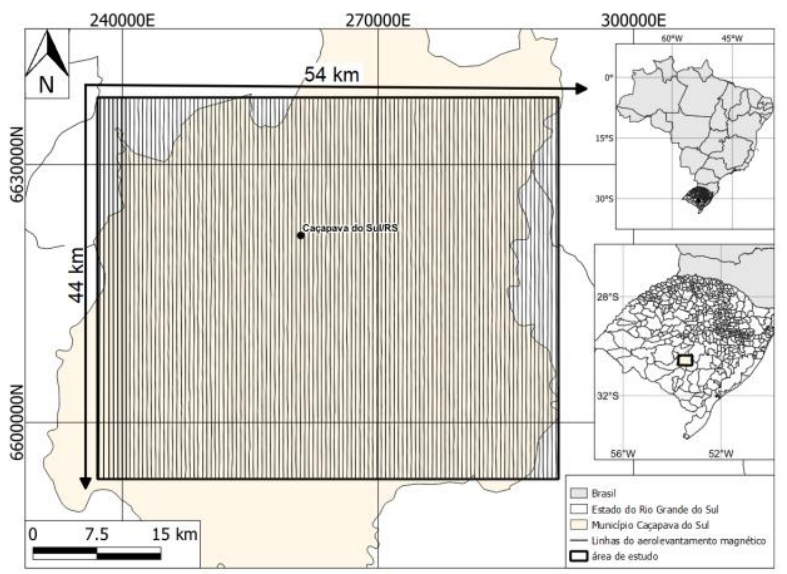

Figura 1 - Localização da área de estudo, limites (polígono) dos dados aerogeofísicos e linhas de voo.

\section{Características da área}

A área definida possui como litotipos principais rochas ígneas, metasedimentares e metamórficas (Borba, 2007). Estas, situam-se no entorno da SGCS que é caracterizada pelas diferentes composições, variando de granítica e granodiorítica (Figueiredo \& Bortoluzzi, 1975). 
Os seus principais minerais acessórios são: alanita, zircão, apatita, titanita, pirita e magnetita.

A sudeste da SGCS situa-se o Granito Santo Ferreira (GSF), descrito por Ribeiro (1970), do qual possui características similares a SGCS.

Como rochas encaixantes e intrudido pela SGCS e pelo GSF se encontra o Complexo Metamórfico Passo Feio (CMPF), representado por rochas metassedimentares (xistos, filitos, mármores, rochas anfiboliticas, metavulcânicas e metavulcanoclásticas) (Nardi \& Bitencourt, 1989).

Alêm destas unidades temos outras que são considerados importantes contribuintes para o arcabouço estrutural da região (Figura 2).

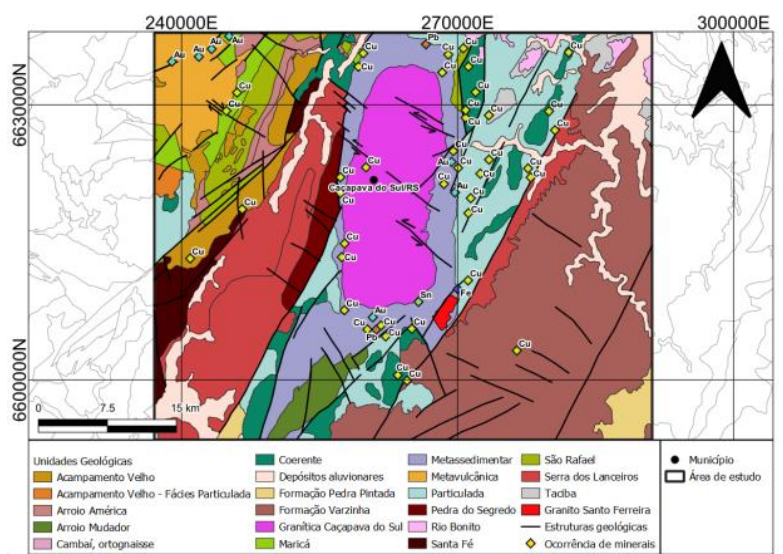

Figura 2- Mapa geológico dos granitos alvos de estudos e suas litologias adjacente. Modificado UFRGS (1998).

No contexto geomorfológico, a região do ESRG sofreu alterações estruturais e de relevo ao longo do tempo geológico. Como consequência, fatores paleomorfoclimáticos, contribuíram significantemente para uma diversificação de ambientes e os contrastes topográficos.

São observadas unidades de depressões, planaltos e planícies com variação altimétrica variando de 44.36 a 453.25 metros (mínimo e máximo respectivamente). As maiores altitudes são representadas por planaltos e os menores pelas planícies. Na Figura 3 são apresentadas as principais ocorrências minerais (CPRM, 2009) e características geomorfológicas da área.

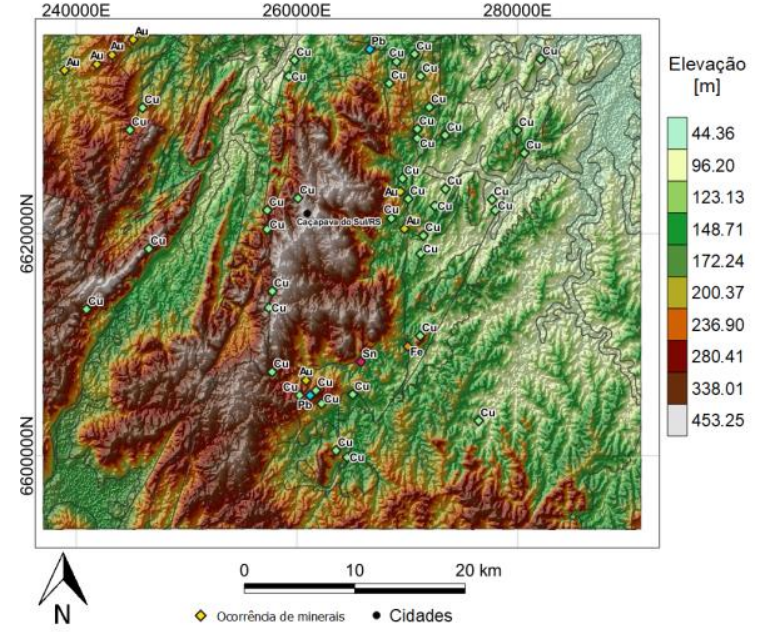

Figura 3 - Mapa de visualização da variação altimétrica da área de estudo TOPODATA (BRASIL, 2008), Área de ocorrência mineral demarcadas (CPRM, 2009).

\section{Metodologia}

Para a realização deste estudo foram definidas 3 etapas principais:

a) Revisão bibliográfica e revisão de dados;

b) Geração da base de dados cartográficos, geológicos e geofísicos;

c) Integração, análise e interpretação.

Para as informações geológicas, mapas e dados de elevação de terreno foram utilizados dados de UFRGS (1998 e TOPODATA BRASIL, 2008). O modelo digital de terreno gerado (MDT) possui uma resolução de 30 metros. A integração de informações foi realizada em um SIG por meio da plataforma de geoprocessamento QGIS versão 13.12.2.

Os dados aeromagnéticos utilizados neste estudo são de domínio público e disponibilizados pelo Serviço Geológico do Brasil (CPRM, 2010). Foram utilizados um total de 106 linhas de voo de direção N-S representados por um pológono de $54 \times 44$ quilômetros. $\mathrm{O}$ espaçamento entre as linhas de voo é de 500 metros e a altura nominal de voo de 100 metros (CPRM, 2010).

$O$ processamento, filtragens e realce dos dados aeromagnéticos foi realizado na plataforma Oasis Montaj. A análise e interpretação dos dados aeromagnéticos apresentados como resultados foi realizada conforme as seguintes etapas e procedimentos:

a) Geração dos dados anômalos de Campo Magnético Anômalo Total (TMI);

b) Aplicação da técnica de Deconvolução de Euler;

c) Separação regional/residual dos dados (TMI);

d) Aplicação dos filtros da primeira Derivada Vertical (DV) e da Amplitude do Sinal Analítico (ASA); 
e) Interpretação dos dados, delineação de lineamentos magnéticos e limites (domínios magnéticos).

Para a geração do grid (modelo interpolado) foi adotado o método de mínima curvatura (Briggs, 1974). O tamanho das células definido foi de 250X250 metros (X e Y).

\section{Resultados e discussões}

As anomalias magnéticas (TMI) obtidas a partir das 106 linhas de voo interpoladas e demais feições de terreno foram integradas em um Sistema de Informações Geográficas (SIG). Obteve-se, a partir desse processamento inicial, os seguintes produtos:

a) Mapa aerogeofísico da área (TMI) e profundidades esperadas estimadas (Figura 4 e 5);

b) Separação Residual/Regional e mapa aerogeofísico (Figuras 6 e 7);

c) Mapa aerogeofísico e resultados da Deconvolução de Euler (Figura 8);

d) Mapa aerogeofísico da Derivada vertical (DV) e Amplitude do Sinal Analítico (ASA) (Figuras 9 e 10);

e) Mapas integrados/Interpretados (Figuras 11, 12 e 13).

\subsection{Mapa das anomalias do Campo Anômalo Total (TMI);}

Os dados de TMl apresentam valores magnéticos variando de -532.93 a $454.21 \mathrm{nT}$ com feições complexas (dipolares) e de difícil identificação quanto a geometria e limites dos corpos causativos (Figura 4).

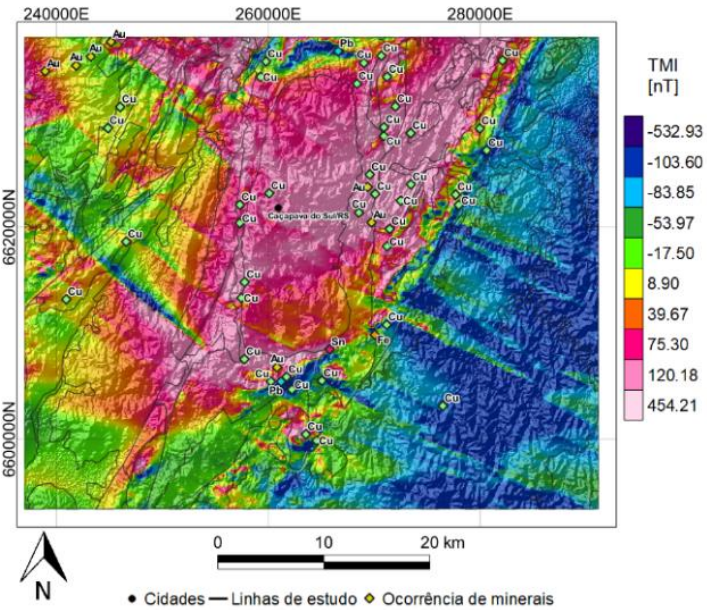

Figura 4 - Mapa aerogeofísico com as anomalias de Campo Anômalo Total (TMI). Sobrepostas, encontram-se as ocorrências minerais conhecidas.

\subsubsection{Separação regional/residual}

Os métodos potenciais possuem variações da faixa de comprimento de onda. Permitem a detecção de fontes profundas (comprimentos longos) e rasas (comprimentos menores). Consequentemente a separação regional/residual dos dados permitem separar parcialmente as respostas superficiais, intermediarias e profundas (Dentith, 2014). A separação regional/residual foi realizada de forma semi-quantitativa a partir da Transformada Rápida de Fourier (FFT) e respectiva geração de um espectro de potência radial dos dados de TMI (Figura 5).

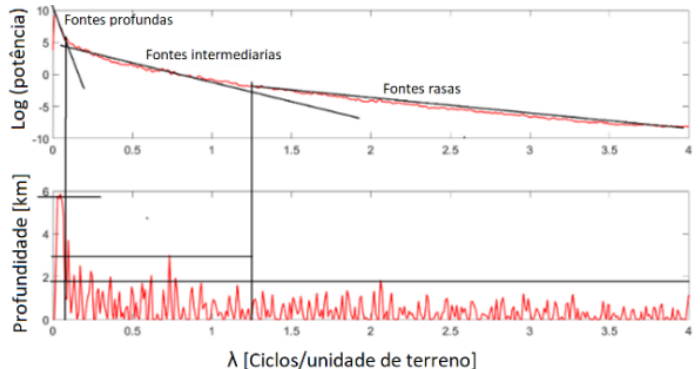

Figura 5 - Espectro de potência radial gerado a partir dos dados do TMI. Linhas pretas delimitam os limites máximos e mínimos (cut-off) de profundidade.

A partir da definição dos valores de corte para aas profundidades aplicou-se um filtro butterworth para a separação residual. Os valores residuais variam de 549.04 a $376.07 \mathrm{nT}$. Observa-se significantes lineações magnéticas demarcadas pelas estruturas mais rasas (Figura 6).

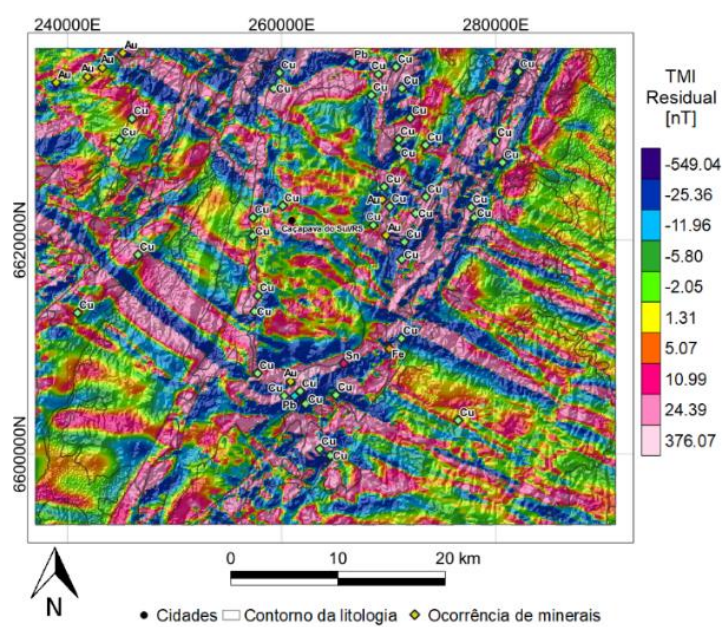

Figura 6 - Mapa aerogeofísico com as anomalias de Campo Magnético Anômalo Total (TMI) submetidos ao filtro butterworth (feições rasas). Sobrepostas, encontramse as ocorrências minerais conhecidas.

Para a separação regional (Figura 7) observa-se valores que variam de -139.85 a $250.27 \mathrm{nT}$. Nota-se a presença de anomalias associadas a fontes/estruturas causativas mais profundas. Estas, são associáveis as litologias na região e a SGCS (Figura 7). 


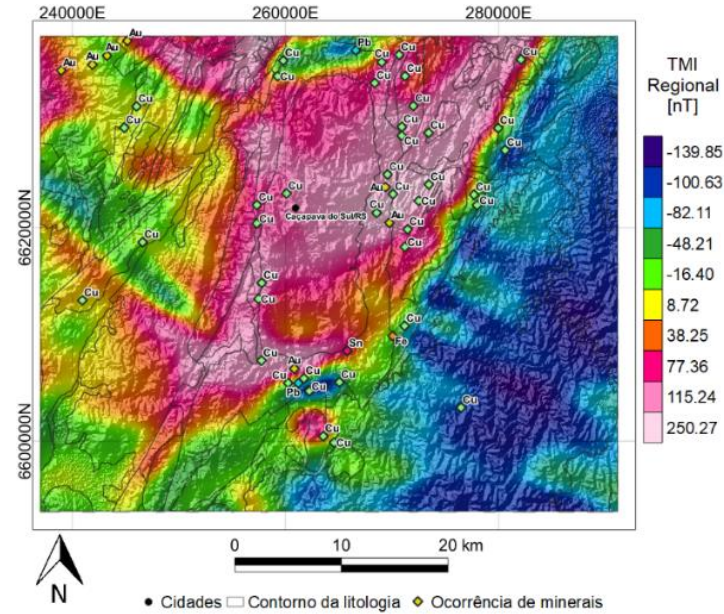

Figura 7 - Mapa aerogeofísico com as anomalias de Campo Magnético Anômalo Total (TMl) submetidos ao filtro butterworth (feições profundas - regional). Sobrepostas, encontram-se as ocorrências minerais conhecidas.

\subsection{Mapas da Deconvolução de Euler 3D}

A aplicação da técnica de Deconvolução de Euler (Thompson, 1982; Reid et al.,1990) é utilizado como uma técnica de realce para interpretação qualitativa, do qual utiliza como principal função o cálculo da profundidade aproximada das anomalias magnéticas. A partir da aplicação da Deconvolução de Euler 3D nos dados de TMI foi possível estimar a profundidade de algumas estruturas causadas por anomalias magnéticas. Como parâmetros de entrada utilizou-se: Tamanho da janela $=10$ e Indice estrutural 0 (zero) (utilizado como uma técnica de realce para interpretação qualitativa, do qual utiliza como principal função o cálculo da profundidade aproximada das anomalias magnéticas) (Thompson, 1982; Reid et al.,1990).

Definiu-se um erro máximo de profundidade de $15 \%$. A partir destes parâmetros foi gerado um mapa conforme apresentado na Figura 8.

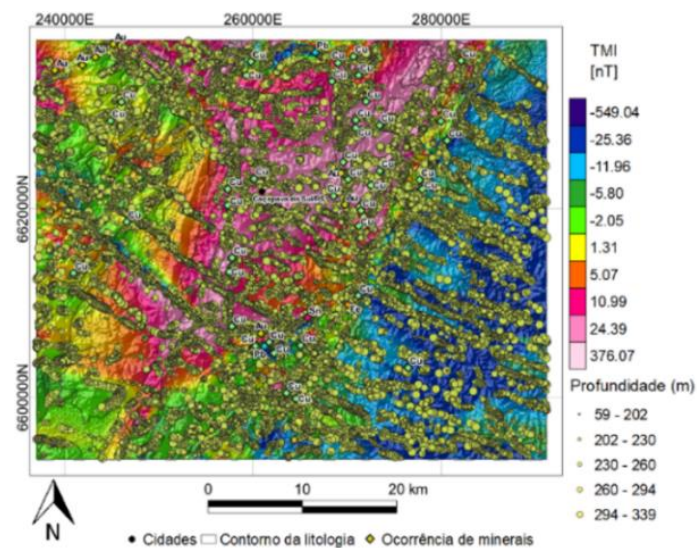

Figura 8 - Mapa das profundidades das fontes anômalas magnéticas (TMI) obtidas a partir da Deconvolução de Euler 3D. Sobrepostas, encontram-se as ocorrências minerais conhecidas.
As anomalias observadas e respectivas fontes de profundidade definidas pela Deconvolução de Euler permitiram a detecção de feições lineares correlacionáveis a fontes magnéticas rasas (separação residual). Estas, variam de 59 a $339 \mathrm{~m}$ de profundidade. Definiu-se um índice estrutural de valor 0 considerando-se a delimitação de falhas e contatos geológicos.

\subsection{Mapas da Derivada Vertical (DV) e Amplitude do Sinal Analítico (ASA)}

A geração da Primeira Derivada Vertical (DV) e da Amplitude do Sinal Analítico (ASA) serviram no auxílio a interpretação e delineação de estruturas lineares e anomalias associadas aos limites e contatos litológicos (Figuras 9 e 10). Estes filtros permitiram a melhor visualização e análise qualitativa das estruturas presentes.

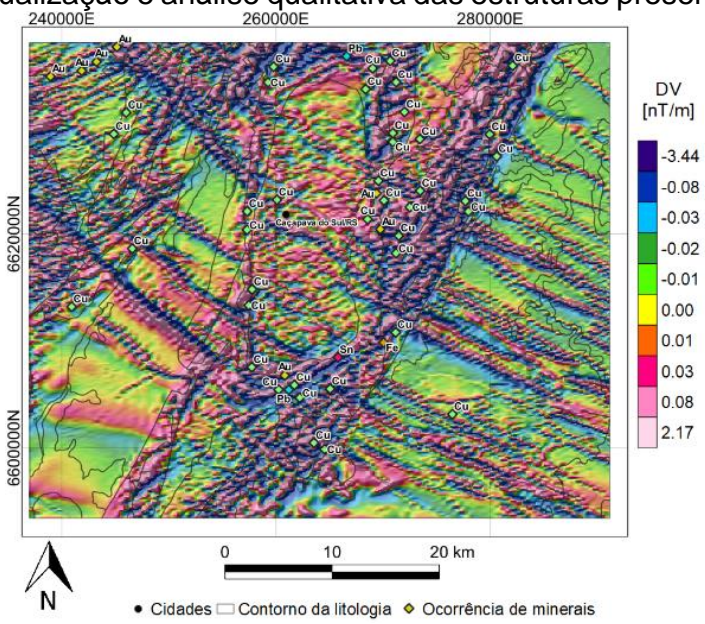

Figura 9 - Mapa aerogeofísico com as anomalias de Campo Magnético Anômalo Total (TMI) submetidas a Primeira Derivada Vertical (DV). Sobrepostas, encontramse as ocorrências minerais conhecidas.

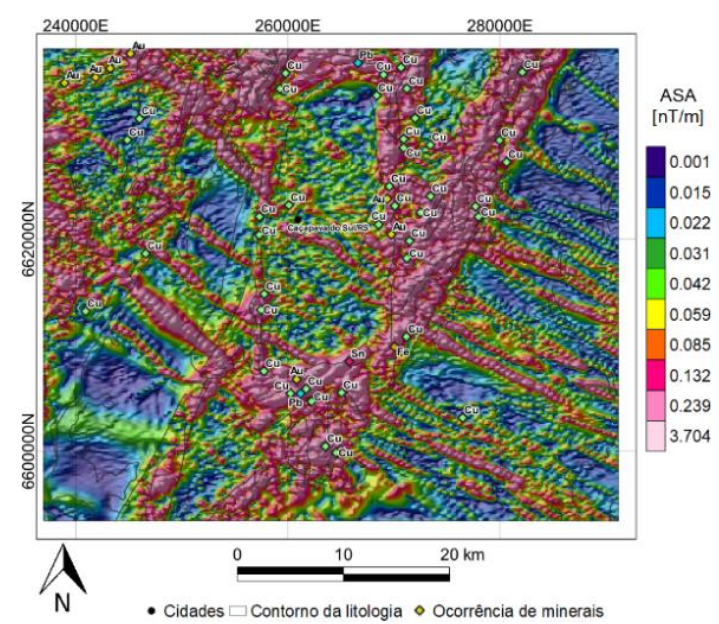

Figura 10 - Mapa aerogeofísico com as anomalias de Campo Magnético Anômalo Total (TMI) submetidas a Amplitude do Sinal Analítico (ASA). Sobrepostas, encontram-se as ocorrências minerais conhecidas.

\subsection{Análises e interpretações}


A partir de analises nos dados dos valores de Campo Magnético Anômalo Total (TMI), valores residuais, regionais filtros, foi possível delimitar seis domínios magnéticos (Figura 11a):

a) Domínio I - associado a SGCS;

b) Domínio II - rochas sedimentares;

c) Domínio III - rochas sedimentares;

d) Domínio IV - rochas vulcanossedimentares e metamórficas;

e) Domínio V - sequências vulcanossedimentares e sedimentares;

f) Domínio VI - rochas ígneas e sedimentares.

A partir das interpretações dos dados obtidos a partir dos mapas de DV e ASA, foi possível a demarcação de lineamentos magnéticos integrados as ocorrências minerais conhecidas separados respectivamente conforme as Figuras 11a e Figura 12a. Para estas feições lineares detectadas, foi gerado um diagrama de roseta com as direções preferenciais e frequências dos lineamentos para a DV e a ASA (Figuras 11b e 12b). Adicionalmente, encontram-se as ocorrências minerais e respectivas direções preferencias nas Figuras 11c e 12c. São observados, para as estruturas detectadas, direções preferenciais de NW - SE e, para as ocorrências minerais conhecidas (Fe, $\mathrm{Cu}, \mathrm{Au}, \mathrm{Sn}$ ), direções preferenciais de NE - SW.

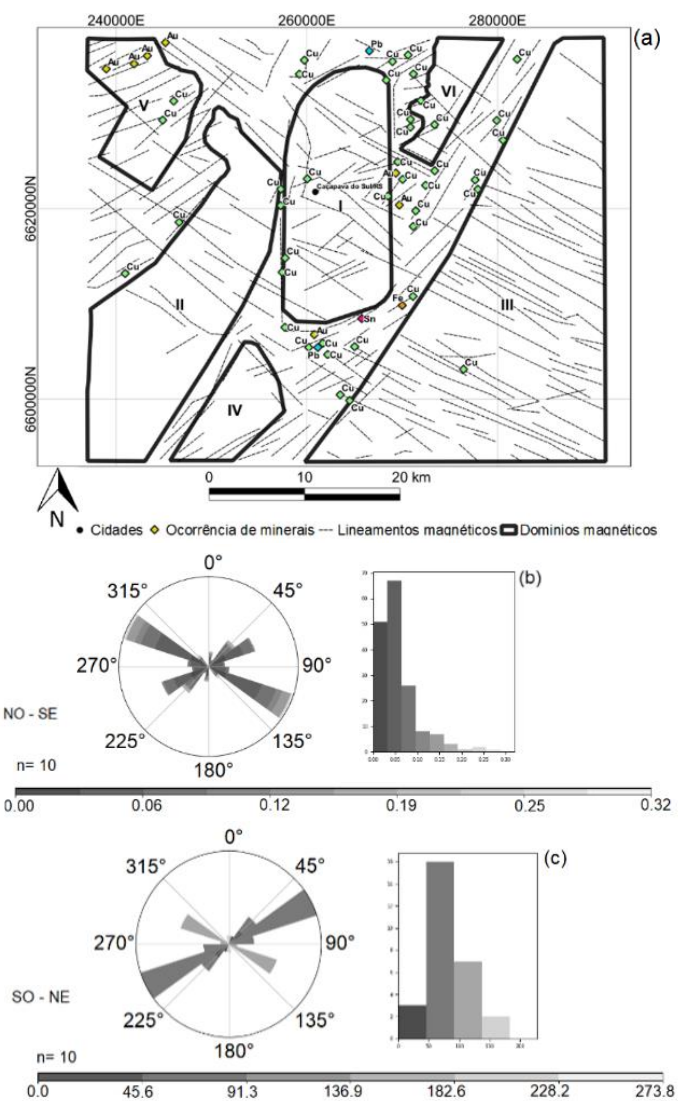

Figura 11 - (a) Mapa geofísico/estrutural dos dados da DV; (b) Diagrama de roseta com as frequências e direções preferenciais dos lineamentos da DV e (c) Diagrama de roseta das frequências e direções preferenciais das ocorrências minerais conhecidas na região.

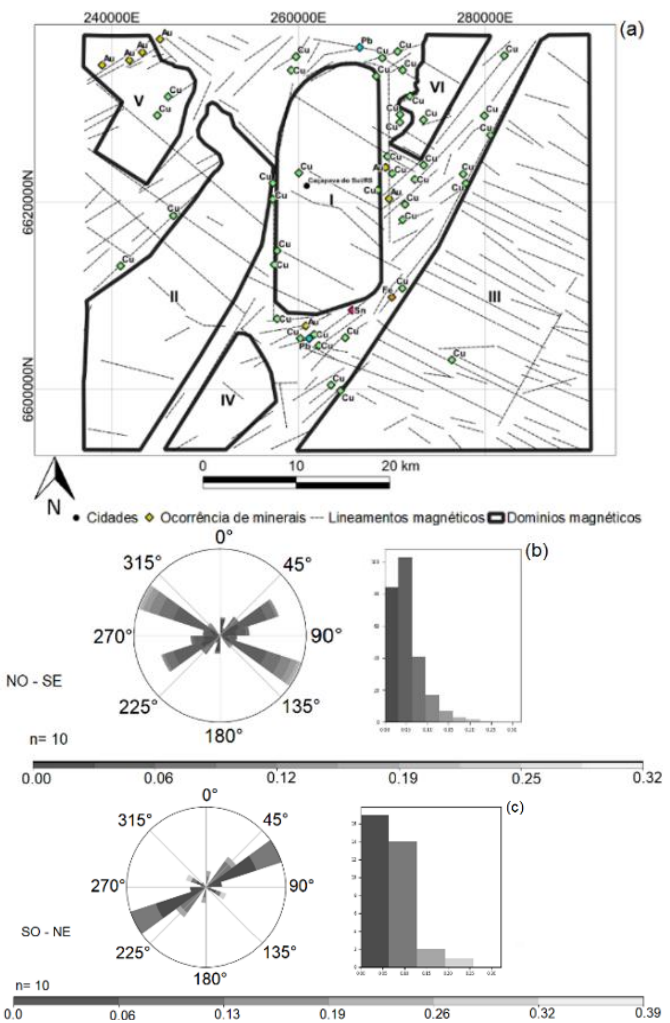

Figura 12 - (a) Mapa geofísico/estrutural dos dados da ASA (b) Diagrama de roseta com as frequências e direções preferenciais dos lineamentos da ASA e (c) Diagrama de roseta das frequências e direções preferenciais das ocorrências minerais conhecidas na região.

A partir dos resultados e análises foi gerado um Mapa litogeofísico conforme apresentado na Figura 13. Foi selecionado para este mapa os valores de maior profundidade obtidos pela Deconvolução de Euler e os respectivos Domínios Magnéticos definidos. Para efeito comparativo com as informações geológicas de superfície, estes resultados foram integrados as estruturas reconhecidas em UFRGS (1988).

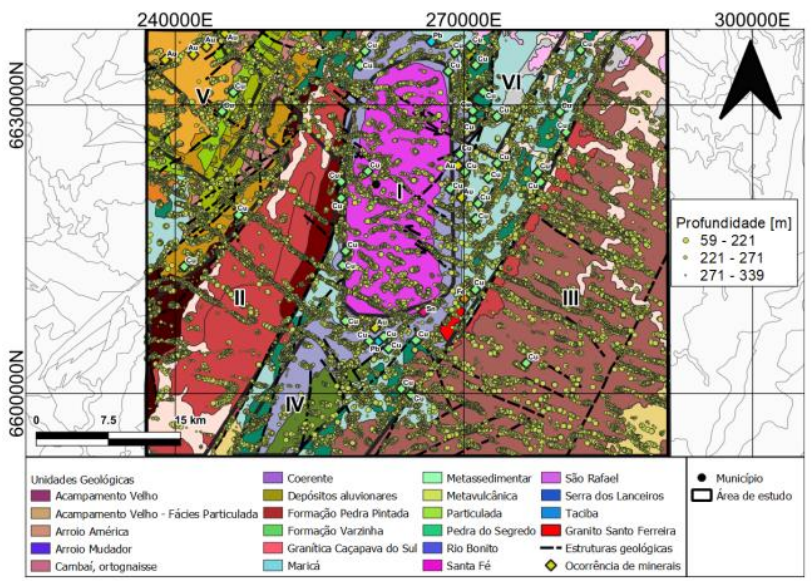


Figura 13 - Mapa litogeofísico gerado a partir das anomalias magnéticas interpretadas, domínios magnéticos e profundidade estimada (Deconvolução de Euler).

\section{Conclusões}

Os resultados deste estudo a partir do processamento e análise das anomalias magnéticas na região da SGCS permitiram se definir domínios magnéticos. Estes, foram gerados a partir da detecção de lineamentos magnéticos observados nos mapas aerogeofísicos. As feições estruturais reconhecidas no estudo consistem em feições não reconhecidas em mapeamentos geológicos prévios. Complementarmente, o uso da Deconvolução de Euler aplicada a estes dados permitiu a definição e caracterização das profundidades estimadas destas feições. Diagramas de roseta (frequência e direção) permitiram a identificação das principais direções destas descontinuidades e associá-las as ocorrências minerais conhecidas a partir de estudos anteriores.

O uso das técnicas de filtros de realce (DV e ASA) e a separação regional/residual colaboraram no realce e delimitação qualitativa dos lineamentos magnéticos contribuindo na determinação de 6 Domínios Magnéticos.

O estudo consiste em importante contribuição no reconhecimento de feições em subsuperfície. Oferece importante conjunto de informações a fim de otimizar os mapeamentos geológicos na região e, no entendimento de estruturas associáveis as ocorrências minerais na região da SGCS. Auxilia, também, na seleção de áreas para futuras aquisições geofísicas terrestres de detalhe em conjunto com a coleta de dados geológicos in situ, como medidas de densidade, susceptibilidade e descrições petrográficas para refinamento dos resultados.

\section{Referências bibliográficas}

ALMEIDA FFM AND HASUI Y. 1984. O Pré-Cambriano do Brasil, São Paulo.

BASEI, M.A.S. 1985. O Cinturão Dom Feliciano em Santa Catarina. São Paulo. 190 p. (Tese de Doutorado, IG-USP). BITENCOURT, M.F. Metamorfitos da região de Caçapava do Sul, RS: Geologia e relações com o corpo granítico. In: Simpósio Sul-Brasileiro de Geologia,1983, Porto Alegre. Anais...Porto Alegre: Sociedade Brasileira de Geologia, 1983.

BRASIL. Instituto Nacional de Pesquisas Espaciais (INPE). Topodata: banco de dados geomorfométricos do Brasil. Variáveis geomorfométricas locais. São José dos Campos, 2008.

BRITO NEVES B.B. \& CORDANI, U.G., 1991. Tectonic evolution of South America during the Late Proterozoic. Precamb.Res., 53: 23-40.

CPRM. Mapa Geodiversidade do Estado do Rio Grande do Sul. 2009

CPRM. Relatório final do levantamento e processamento dos dados Magnetométricos e gamaespectrométricos. v.1. 2010.

CPRM. Sobre o GeoSGB. Disponível em: < http://geosgb.cprm.gov.br/>. Acesso em: 01 de fevereiro de 2020.
DENTITH, M.; MUDGE, S.T. Geophysics for the mineral exploration geoscientist. Cambridge University Press, 2014.

Equipe de desenvolvimento do QGIS, <2020>. Sistema de Informação Geográfica QGIS. Projeto da Fundação Geoespacial de Código Aberto. http://qgis.osgeo.org.

FIGUEIREDO JR., A. G. (1975) Geologia os depósitos calcáreos biodetríticos da plataforma continental do Rio Grande do Sul. Porto Alegre, UFRGS: Dissertação Mestrado.

FRAGOSO CÉSAR, A.R.S. 1980. O Cráton Rio de la Plata e o Cinturão Dom Feliciano no Escudo Uruguaio-SulRiograndense. In: CONGR. BRAS. GEOL, 31. Curutiba, 1980. Anais... Curitiba, SBG. v. 5, p. 2879-2892.

FRAGOSO CESAR, A.R.S.; RACHADO, R. 6 GOMEZ, CR - 1987 - Observações sobre o Cinturão Doa Feliciano no Escudo Uruguaio e correlações coei o Escudo do rio Grande do Sul. III simp. Sul-Bras.Geol.. Atas. 2:791-809. Curibita.

FRAGOSO, A.R.S. 1991. Tectônica de Placas no Ciclo Brasiliano: As Orogenias dos Cinturões Dom Feliciano e Ribeira no Rio Grande do Sul. São Paulo. 367 p. (Tese de Doutorado, IG-USP).

HARTMANN, L. A.; Chemale Jr., F.; Philipp, R. P., 2007. Evolução Geotectônica do Rio Grande do Sul no PréCambriano. In: Iannuzzi, R. \& Frantz, J.C. (Ed.): 50 ANOS de Geologia: Instituto de Geociências. Contribuições, p. 97-123

NARDI, L.V.S. \& BITENCOURT, MF. Geologia, petrologia e geoquímica do Complexo Granítico de Caçapava do Sul, RS. Revista Brasileira de Geociências, 153-169. 1989.

PAVLIS, N. K., Holmes, S. A., Kenyon, S. C., and Factor, J. K. (2012), the development and evaluation of the Earth Gravitational Model 2008 (EGM2008), J. Geophys. Res., 117, B04406, doi: 10.1029/2011JB008916.

REID, A.B., Allsop, J.M., Granser, H., Millett, A.J. \& Somerton, I.W., 1990. Magnetic interpretation in three dimensions using Euler deconvolution, Geophysics, v $55,80-91$.

REMUS M.V.D. Metalogênese dos depósitos hidrotermais de metais base e Au do Ciclo Brasiliano do Bloco São Gabriel, RS. Tese de Doutorado. Universidade Federal do Rio Grande do Sul- URGS, 170P, 1999.

RIBEIRO, M. Geologia da Folha de Bom Jardim, Rio Grande do Sul, Brasil. Bol. Div. Geol. Min. Bras. Rio de Janeiro: DNPM, v.247, p.1-142, 1970.

THOMPSON, D.T., 1982, EULDPH: A new technique for making computer-assisted depth estimates from magnetic data, Geophysics, v 47, 31-37. .

Universidade Federal do Rio Grande do Sul (UFRGS). 1998. Mapeamento Geológico 1:25000: Projeto Caçapava do Sul. Porto Alegre. 